SLAC-PUB-8625

BABAR-PROC-00/16

LPNHE-00-05

hep-ex/0009051

September, 2000

\title{
Measurements of $B^{0}$ and $B^{ \pm}$lifetimes and $B^{0}-\bar{B}^{0}$ mixing with fully reconstructed $B$ decays in BABAR
}

\author{
Fernando Martínez-Vidal \\ LPHNE, IN2P3-CNRS/Universités Paris $6 \& 7$, France \\ E-mail: martinef@SLAC.Stanford.EDU \\ (for the $B A B A R$ Collaboration)
}

\begin{abstract}
Time-dependent $B^{0} \bar{B}^{0}$ flavor oscillations and $B^{0}$ and $B^{+}$lifetimes are studied in a sample of fully reconstructed $B$ mesons collected with the BABAR detector, running at the PEP-II asymmetric $e^{+} e^{-} B$ Factory with center-of-mass energies near the $\Upsilon(4 S)$ resonance. This is the first time that time-dependent mixing and lifetime measurements have been performed at $\Upsilon(4 S)$ energies.
\end{abstract}

Contributed to the Proceedings of the $30^{\text {th }}$ International Conference on High Energy Physics, 7/27/2000-8/2/2000, Osaka, Japan

Stanford Linear Accelerator Center, Stanford University, Stanford, CA 94309

Work supported in part by Department of Energy contract DE-AC03-76SF00515. 


\section{Introduction}

Preliminary measurements of time-dependent $B^{0} \bar{B}^{0}$ flavor oscillations and $B^{0}$ and $B^{+}$lifetimes have been performed with the BABAR detector. These analyses exploit the copious production of $B$ meson pairs in $\Upsilon(4 S)$ decays, produced by asymmetric $e^{+} e^{-}$collisions at the PEP-II $B$ Factory at SLAC. These measurements can be used to test theoretical models of heavy quark decay and to constrain the Unitarity Triangle (via the sensitivity to the value of the Cabibbo-Kobayashi-Maskawa matrix [1] element $\left.V_{t d}\right)$. The data set, collected from January to June, 2000, has an integrated luminosity of $8.9 \mathrm{fb}^{-1}$ on the $\Upsilon(4 S)$ resonance and 0.8 $\mathrm{fb}^{-1}$ collected $40 \mathrm{MeV}$ below the $B \bar{B}$ threshold. This corresponds to about $(10.1 \pm 0.4) \times 10^{6}$ produced $B \bar{B}$ pairs. The resolution function and mistag rates determined from data in the analyses described here are also used in $C P$ asymmetry measurements [2].

\section{Experimental Method}

The BABAR detector is described in detail elsewhere [3]. The analyses described here use all the detector capabilities, including high resolution tracking and calorimetry, particle identification and vertexing.

At PEP-II the $B$ meson pairs produced in the decay of the $\Upsilon(4 S)$ resonance are moving in the lab frame along the beam axis ( $z$ direction) with a Lorentz boost of $\beta_{z} \gamma=0.56$. One $B\left(B_{\mathrm{REC}}\right)$ is fully reconstructed in an all-hadronic $\left(B^{0} \rightarrow D^{(*)-} \pi^{+}, D^{(*)-} \rho^{+}, D^{(*)-} a_{1}^{+}, J / \psi K^{* 0}\right.$ and $\left.B^{-} \rightarrow D^{(*) 0} \pi^{-}, J / \psi K^{-}, \psi(2 S) K^{-}\right)$or semileptonic decay mode $\left(B^{0} \rightarrow D^{*-} \ell^{+} \nu\right) *$. A total of about 2600 neutral, and a similar number of charged, $B$ candidates is reconstructed in hadronic decay modes, with an average purity close to $90 \%$. The background is mainly combinatorial. About $7500 \mathrm{~B}^{0}$ 's are reconstructed in semileptonic modes, with an average purity of $\sim 84 \%$. Backgrounds to the semileptonic mode are due to combinatorics, fake leptons, $c \bar{c}$ events, and charged $B$ decays from $B^{-} \rightarrow D^{*+}(n \pi) l^{-} \nu$. Fig. 2 shows the beamenergy substituted $B$ mass $\left(m_{\mathrm{ES}}\right)$ distributions for the hadronic sample (left) and the $D^{*}-D^{0}$ mass distribution for the semileptonic sample (right) $[4,5]$.

The separation between the two $B$ vertices along the boost direction, $\Delta z=z_{\mathrm{REC}}-z_{\mathrm{TAG}}$, is measured and used to estimate the decay time difference, $\Delta t \approx \Delta z / \beta_{z} \gamma c$. The $B_{\mathrm{TAG}}$ vertex is determined via an inclusive procedure applied to all tracks not associated with the $B_{\text {REC }}$ meson [4]. The typical separation between the two vertices is $\Delta z=\beta_{z} \gamma c \tau_{B} \approx 260 \mu \mathrm{m}$, to be compared to the experimental resolution $\sim 110 \mu \mathrm{m}$. The $\Delta t$ resolution is dominated by the precision on the $B_{\mathrm{TAG}}$ vertex, and has little dependence on the decay mode of the $B_{\mathrm{REC}}$. An event-by-event $\Delta z$ resolution is computed and modified to fit the data by convolution with three Gaussians, core, tail and outlier. Most of the events, $\sim 70 \%$, are in the core Gaussian, with $\sigma \sim 0.6$ ps.

\footnotetext{
*Throughout this paper, conjugate modes are implied.
} 

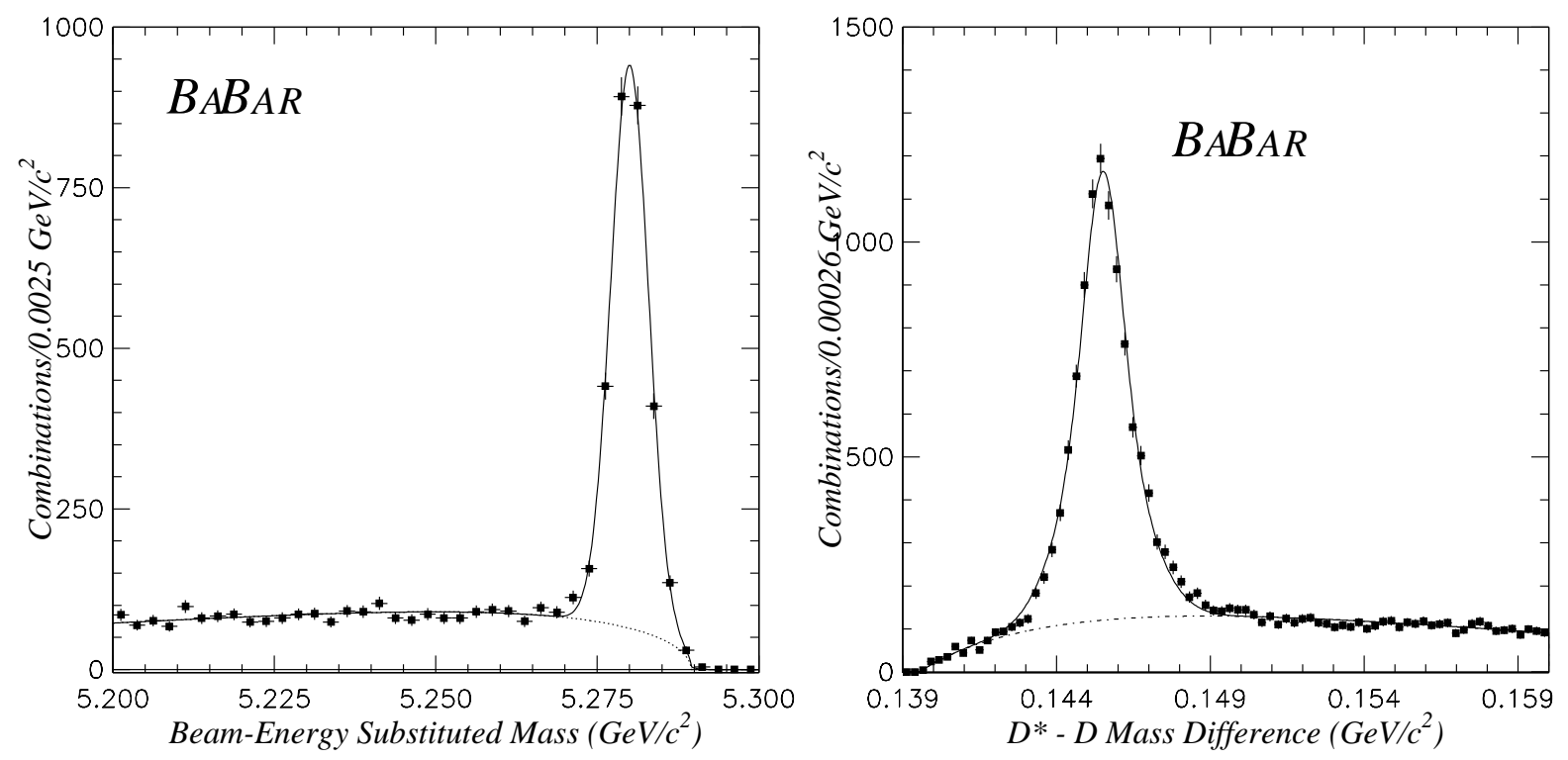

Figure 1: Left: Beam-energy substituted mass distribution $\left(m_{\mathrm{ES}}\right)$ for all the hadronic $B^{0}$ modes. Right: $D^{*}-D^{0}$ mass difference distribution for the $B^{0} \rightarrow D^{*-} \ell^{+} \nu$ sample.

\section{Lifetime Measurements}

The $B^{0}$ and $B^{+}$lifetimes are extracted from a simultaneous unbinned maximum likelihood fit to the $\Delta t$ distributions of the signal candidates, assuming a common resolution function. Only hadronic decays from a subsample of $7.4 \mathrm{fb}^{-1}$ integrated luminosity (on-resonance) have been used. An empirical description of the $\Delta t$ background shape is assumed, using $m_{\mathrm{ES}}$ sidebands with independent parameters for neutral and charged mesons. Fig. 2 shows the $\Delta t$ distributions with the fit result superimposed. Table 1 summarizes the contributions to the total error (see [4] for details).

\section{Time-dependent $B^{0} \bar{B}^{0}$ mixing}

A time-dependent $B^{0} \bar{B}^{0}$ mixing measurement requires the determination of the flavor of both $B$ 's. The $B_{\mathrm{REC}}$ flavor is known if it has been correctly reconstructed, and the flavor of the $B_{\mathrm{TAG}}$ is determined by exploiting the correlation between the flavor of the $B_{\mathrm{TAG}}$ meson and the charge of its decay products [5]. If there is an identified lepton its charge is used; otherwise the summed charge of identified kaons provides the tag. An event with no tagging leptons or kaons can still be tagged by use of a neural net that exploits the flavor information carried by other decay products, such as soft leptons from charm semileptonic decays and soft pions from $D^{*}$ decays.

The effective flavor tagging efficiency is given by $Q=\sum_{i} \epsilon_{i}\left(1-2 w_{i}\right)^{2}$ where the sum is over tagging categories, each characterized by a tagging efficiency $\epsilon_{i}$ and a probability to 

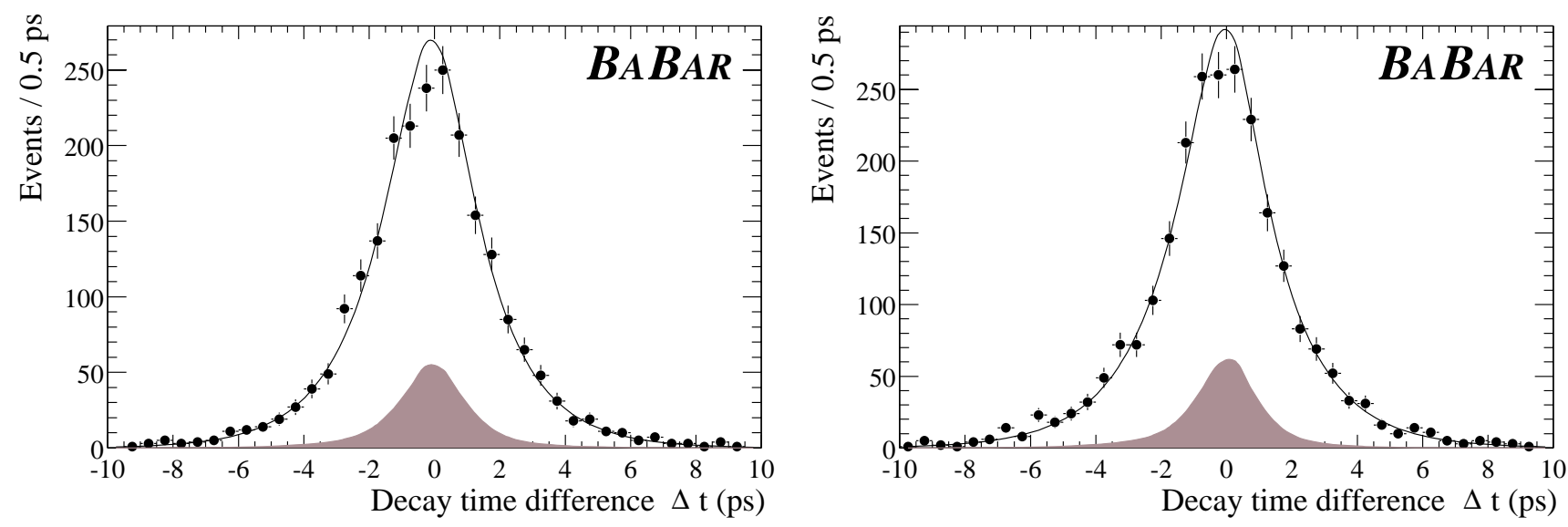

Figure 2: $\Delta t$ distributions for $B^{0} / \bar{B}^{0}$ (left) and $B^{+} / B^{-}$(right) candidates in the signal region $\left(m_{\mathrm{ES}}>5.27 \mathrm{GeV} / \mathrm{c}^{2}\right)$. The result of the lifetime fit is superimposed. The background is shown by the hatched area.

mis-identify the $B$ flavor, $w_{i}$. $Q$ is related to the statistical significance of the measurement $\left(1 / \sigma_{\text {stat }}^{2} \sim N_{B_{\mathrm{TAG}}} Q\right)$.

From the time-dependent rate of mixed $\left(N_{\text {mix }}\right)$ and unmixed $\left(N_{\text {unmix }}\right)$ events, the mixing asymmetry $a(\Delta t)=\left(N_{\text {unmix }}-N_{\text {mix }}\right) /\left(N_{\text {unmix }}+N_{\text {mix }}\right)$ is calculated as a function of $\Delta t$ and fit to the expected cosine distribution,

$$
a(\Delta t) \propto(1-2 w) \cos \Delta m_{d} \Delta t \otimes \mathcal{R}(\Delta t \mid \hat{a}),
$$

where $\hat{a}$ are the parameters of the resolution function [5]. A simultaneous unbinned likelihood fit to all the tagging categories, assuming a common resolution function, allows the determination of both $\Delta m_{d}$ and the mistag rates, $w_{i}$. An empirical description of the backgrounds is determined by fitting to background control samples taken from data, allowing for the following components: i) zero lifetime, ii) non-zero lifetime with no mixing, iii) non-zero lifetime with mixing (only for semileptonic decays). Fig. 3 shows the $a(\Delta t)$ distributions with the fit result superimposed. Table 2 summarizes all the contributions to the total error (see [5] for details).

\section{Results}

The preliminary results for the $B$ meson lifetimes are

$$
\begin{aligned}
& \tau_{B^{0}}=1.506 \pm 0.052(\text { stat }) \pm 0.029(\text { syst }) \mathrm{ps} \\
& \tau_{B^{+}}=1.602 \pm 0.049(\text { stat }) \pm 0.035(\text { syst }) \mathrm{ps}
\end{aligned}
$$

and for their ratio is

$$
\tau_{B^{+}} / \tau_{B^{0}}=1.065 \pm 0.044 \text { (stat) } \pm 0.021 \text { (syst). }
$$


Table 1: Summary of uncertainties for the $B$ Lifetime measurements.

\begin{tabular}{|c|c|c|c|}
\hline Source & $\sigma\left(\tau_{B}^{0}\right)$ & $\sigma\left(\tau_{B}^{+}\right)$ & $\sigma\left(\tau_{B}^{+} / \tau_{B}^{0}\right)$ \\
\hline Data statistics & 0.052 & 0.049 & 0.044 \\
\hline MC statistics & 0.016 & 0.014 & 0.014 \\
$\Delta t$ resolution & 0.007 & 0.007 & 0.008 \\
$\Delta z$ outliers & 0.016 & 0.020 & 0.005 \\
alignment & - & - & - \\
$z$ scale & 0.015 & 0.016 & - \\
boost & 0.006 & 0.006 & - \\
signal probability & 0.003 & 0.002 & 0.005 \\
background & 0.005 & 0.017 & 0.011 \\
\hline Total systematics & 0.029 & 0.035 & 0.021 \\
\hline
\end{tabular}

Table 2: Break-down of uncertainties for the $B$ Mixing measurements.

\begin{tabular}{|c|c|c|}
\hline Source & $\begin{array}{c}\sigma\left(\Delta m_{d}\right) \\
\text { hadronic }\end{array}$ & $\begin{array}{c}\sigma\left(\Delta m_{d}\right) \\
\text { semileptonic }\end{array}$ \\
\hline Data statistics & 0.031 & 0.020 \\
\hline MC statistics & 0.011 & 0.009 \\
$\Delta t$ resolution & 0.011 & 0.012 \\
background $\Delta t$ & 0.002 & 0.002 \\
background dilutions & - & 0.006 \\
background fractions & 0.004 & 0.006 \\
$B^{+}$background & - & 0.010 \\
$B^{0}$ Lifetime & 0.005 & 0.006 \\
$z$ scale & 0.005 & 0.005 \\
boost & 0.003 & 0.003 \\
\hline Total systematics & 0.018 & 0.022 \\
\hline
\end{tabular}

From the hadronic $B^{0}$ sample we measure the $B^{0} \bar{B}^{0}$ oscillation frequency:

$$
\Delta m_{d}=0.516 \pm 0.031 \text { (stat) } \pm 0.018 \text { (syst) } \hbar \mathrm{ps}^{-1}
$$

and from the $D^{*-} \ell^{+} \nu$ sample the result is

$$
\Delta m_{d}=0.508 \pm 0.020 \text { (stat) } \pm 0.022(\text { syst }) \hbar \mathrm{ps}^{-1} \text {. }
$$

Combining the two $\Delta m_{d}$ results, we obtain the preliminary result:

$$
\Delta m_{d}=0.512 \pm 0.017(\text { stat }) \pm 0.022(\text { syst }) \hbar \mathrm{ps}^{-1} .
$$

The mistag rates and $\Delta t$ resolution function extracted from these fits are used in the BABAR $C P$ violation asymmetry analysis [2]. The effective flavor tagging efficiency is found to be $Q \approx 28 \%$. 

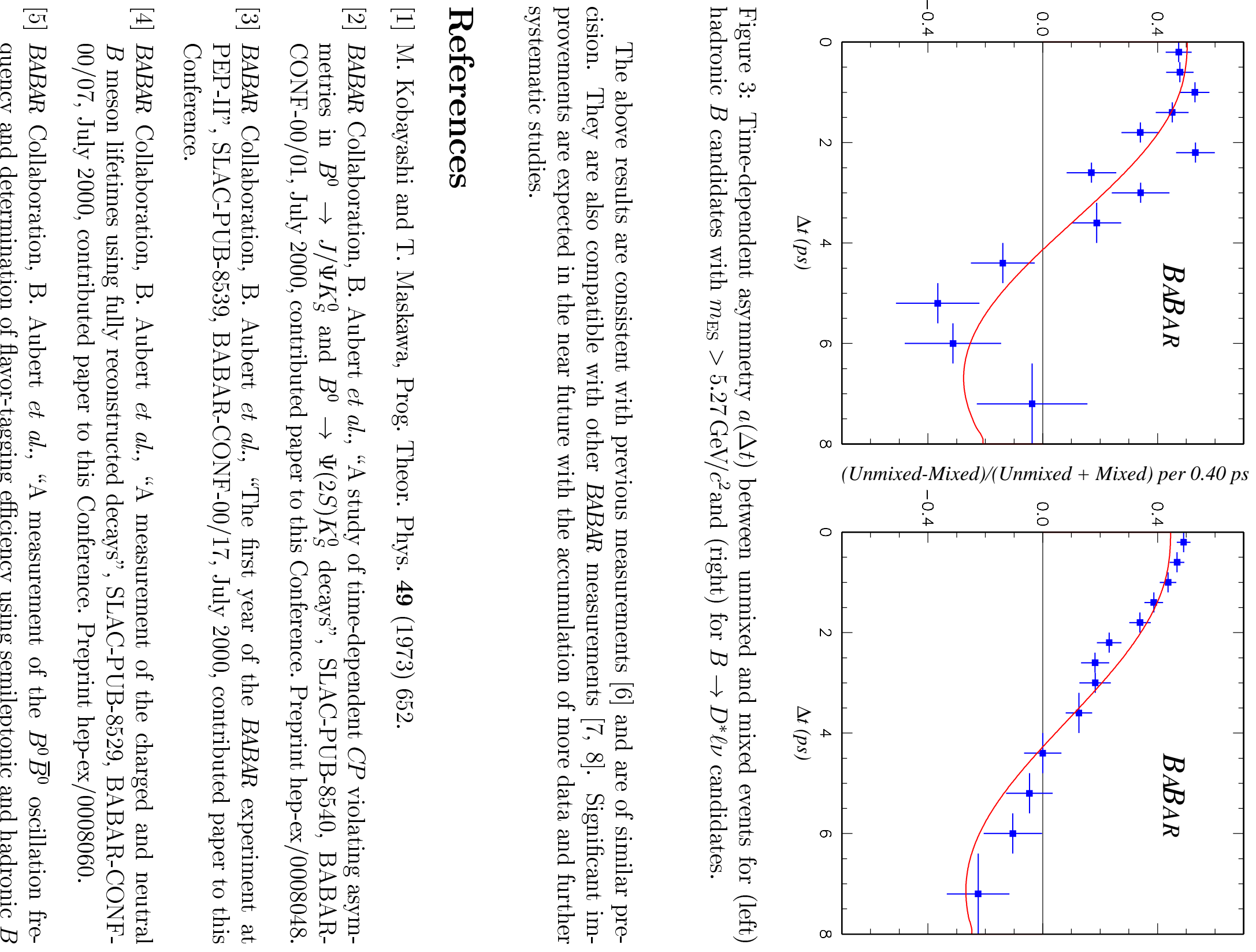
decays", SLAC-PUB-8530, BABAR-CONF-00/08, July 2000, contributed paper to this Conference. Preprint hep-ex/0008052.

[6] D.E. Groom, et al., Eur. Phys. Jour. C 15(2000) 1.

[7] B. Aubert et al., "Measurement of the $B^{0}$ meson properties using partially reconstructed $B^{0}$ to $D^{*-} \pi^{+}$and $B^{0}$ to $D^{*-} \ell^{+} \nu$ decays with the BABAR detector", SLAC-PUB-8531, BABAR-CONF-00/09, July 2000, contributed paper to this Conference. Preprint hepex/0008053.

[8] B. Aubert et al., "Measurement of the time dependence of $B^{0} \bar{B}^{0}$ oscillations using inclusive dilepton events", SLAC-PUB-8532, BABAR-CONF-00/10, July 2000, contributed paper to this Conference. Preprint hep-ex/0008054. 